\title{
Propuesta Para Un diseÑo DE Distribución EN Planta EN EL ÁREA DE SEPARADO PARA UNA EMPRESA DE ALIMENTOS CÁRNICOS, EVALUADA MEDIANTE UNA HERRAMIENTA DE SIMULACIÓN - FLEXSIM
}

\author{
Proposal For a PLANT LAYOUT IN THE SEPARATION \\ AREA OF MEAT FOOD,EVALUATED BY \\ A SIMUlation TOOL - FLEXSIM
}

\author{
Helmer Paz-Orozco ${ }^{1}$, Julian Cañar-Truque ${ }^{2}$, Luz Adriana Plazas-Pemberthy ${ }^{3}$, \\ Hector Angulo-Sinisterra ${ }^{4}$ \\ Corporación Universitaria Comfacauca, Facultad de Ingeniería, Popayán-Cauca, Colombia \\ Fundación Centro Colombiano de Estudios Profesionales, Facultad de Ingeniería Santiago de Cali, Cali, Colombia
}

Recibido: 23/03/2018 • Aprobado: 12/04/2018

\section{RESUMEN}

La presente investigación presenta un nuevo diseño de distribución en planta (Layout) para el proceso de separado en la empresa de Alimentos Cárnicos S.A.S, una empresa del grupo empresarial Nutresa dedicada a la producción y comercialización de embutidos procesados. La investigación consistió en el diagnóstico, análisis e identificación de oportunidades de mejora en la distribución en planta del proceso de separado de chorizos calibre 26 y en la propuesta de una alternativa de mejoramiento de distribución en planta aplicando la metodología de relación de actividades, con el objetivo de mejorar la efectividad de las operaciones, reduciendo tiempos y costos para la empresa. La metodología fue evaluada en simulación mediante el software Flexsim; una herramienta de simulación que permite evaluar los procesos productivos industriales. En el proceso de simulación se valoraron las variables de tiempo de ocupación, tiempo de inactividad y distancias que recorren los operarios en cuanto a la relación Hombre - Máquina. La investigación tuvo como finalidad optimizar la distribución de las maquinas, recursos humanos, materiales y servicios auxiliares. Finalmente, el estudio propone un escenario de distribución en planta donde se mejoran considerablemente las distancias recorridas por los operarios y los porcentajes de inactividad de máquinas y trabajadores, proyectando una disminución de cuellos de botella, la optimización de la mano de obra, la reducción del riesgo para la salud y el aumento de la seguridad del recurso humano.

Palabras clave: distribución de planta, diseño, flexsim, simulación.

\footnotetext{
${ }^{1}$ hpaz@unicomfacauca.edu.co, orcid.org/0000-0003-4661-8538

2 juliancanar@unicomfacauca.edu.co, orcid.org/0000-0003-0871-2195

3 lplazas@unicomfacauca.edu.co, orcid.org/0000-0001-7301-9683

${ }^{4}$ hector.angulo@cecep.edu.co, orcid.org/0000-0003-4402-7275
} 


\section{Abstract}

The following research presents a new plant layout for the separation process of Alimentos Cárnicos S.A.S, a company of Nutresa business group, which is dedicated to the production and commercialization of processed sausages. The research consisted in the diagnosis, analysis and identification of opportunities for improvement in the plant layout of the separation process for chorizos 26. It was proposed an improvement alternative of plant layout applying the methodology of activities relation with the aim of improving the effectiveness of operations, in order to reduce time and costs for the company. The methodology was evaluated in the simulation software Flexsim; a simulation tool that allows to evaluate industrial production processes. In the simulation process, it was evaluated the occupation time, inactivity time and travel distances regarding the Man - Machine relationship. The purpose of the research was to optimize the distribution of machines, human resources, materials and auxiliary services. Finally, a plant layout scenario was proposed, where the traveled distances and the inactivity of machines and workers have improved considerably, projecting a decrease of bottlenecks, optimization of the workforce, reducing of the risk to health and increasing the safety of human resources.

Key words: design, flexsim, layout, simulation.

\section{INTRODUCCIÓN}

Las actividades industriales de las empresas se rigen por las condiciones del mercado, que cada vez es más selectivo y exigente. Es por ello que todos los aspectos del proceso productivo deben ser eficientes y garantizar la subsistencia de las empresas, especialmente de aquellas en la industria de alimentos (Collazos Valencia, 2013) (Khuriyati, Sukartiko, \& Kartikasari, 2016). Una de las fases que debe analizarse y diseñarse cuidadosamente es la distribución de las actividades del proceso productivo en la planta, ya que influencia directamente los costos de producción y por tanto afecta la competitividad de la empresa. (Diego-Más, 2006).

La planta de producción de Alimentos Cárnicos S.A.S se dedica a la producción de alimentos en la línea de embutidos cárnicos y está ubicada en la Zona Franca del norte del Cauca, Colombia. Esta planta produce 19 referencias distintas de salchichas y chorizos en altos volúmenes, como registra su producción para el último semestre de 2016, que fue de 10,236 toneladas. (Grupo Empresarial Nutresa S.A, 2010). Con el objetivo de aumentar la eficiencia en la planta desde la gestión del recurso humano, se planteó una revisión, modelamiento y simulación del proceso de separado, con el fin de proponer modificaciones en la distribución y orden de los operarios y máquinas que signifiquen una mejora en tiempos y costos para la empresa. (Khiewwijit, Temmink, Rijnaarts, \& Keesman, 2015)

Para este estudio se priorizo el producto chorizo campesino calibre 26, que presenta mayor participación en el mercado, con el $12.70 \%$ que equivale al 1,300 toneladas aproximadamente (Alimentos Carni$\cos$ S.A.S, 2016) .

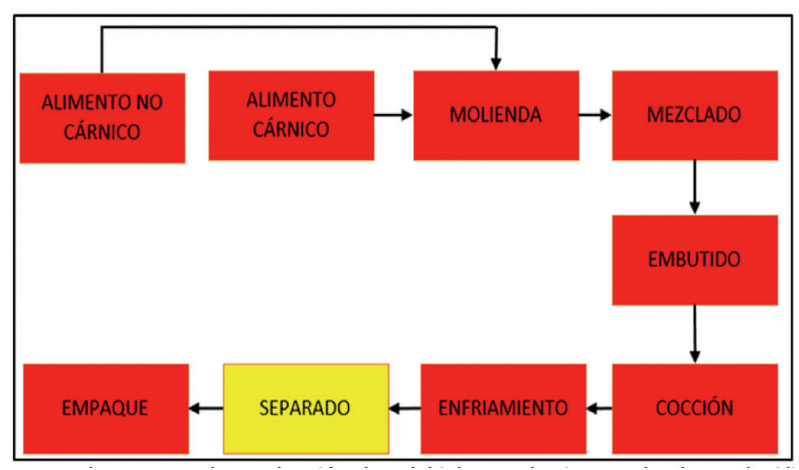

Fig. 1 Diagrama de proceso de producción de salchichas y chorizos en la planta de Alimentos Cárnicos S.A.S

Fuente: autores 
El proceso de separado, representado en la Figura 1, inicia cuando los productos salen del área de enfriamiento y son almacenados en carros para embutidos. Los operarios 1 y 2 descargan los carros y depositan los productos en las mesas. Los operarios 3, 4, 5 y 6 alimentan cada una de las maquinas separadoras. Una vez el producto se separa en unidades individuales, los operarios 7 y 8 lo reciben en canastillas plásticas, que son tomadas y depositadas en una banda transportadora hasta el área de empaque. El proceso de separado termina cuando el operario deposita la canastilla plástica en la banda transportadora, en la Figura 2 se presenta un esquema del proceso.

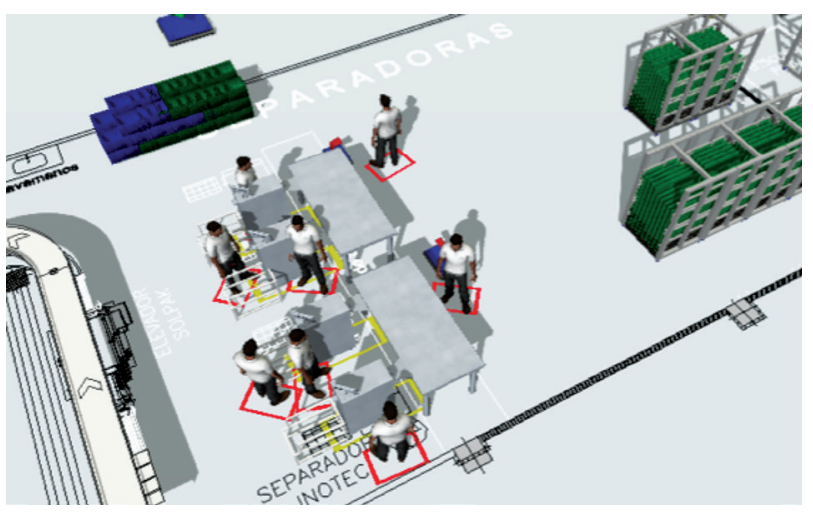

Fig. 2 Proceso de separado en la planta

La presente investigación, propone una mejora del diseño de distribución en planta (Layout) en el área de separado (Caputo, Pelagagge, Palumbo, \& Salini, 2015), por medio de la metodología de relación de actividades ${ }^{6}$ (Vargas, Vargas, \& Franco, 2018), analizando los diferentes procesos productivos, productos, máquinas y operarios que intervienen en el área de separado, utilizando una herramienta especializada en diseño (AutoCAD) con el fin de ser evaluados por mediante del software Flexsim, simulador de eventos discretos que permite modelar, analizar, visualizar y optimizar el proceso industrial (Practicas de sistemas de fabricación., 2012).
"La distribución en planta es un fundamento de la industria. Determina la eficiencia y, en algunos casos, la supervivencia de una empresa" (Muther, 1981).

Para el nuevo diseño en el área de separado de la empresa Alimentos Cárnicos S.A.S, se aplicó la metodología de relación de actividades planteada y definida por Richard Muther, como "el acto de planificar, el ordenamiento óptimo de las actividades industriales, incluyendo personal, equipo, almacenes, sistemas de manutención de materiales, y todos los otros servicios anexos que sean necesarios para diseñar de la mejor manera posible la estructura que contenga estas actividades" (Muther, 1981).

En la metodología mencionada se muestran las relaciones de cada departamento y permite realizar un estudio de los flujos productivos determinantes para la distribución (flujo de materiales, información, operadores, etc.), desarrollando una matriz de relaciones en la que se asigna por pares de instalaciones una etiqueta de acuerdo con la razón de cercanía que refleja la mayor o menor necesidad de situar próximas secciones de dicho par (Heidy Mejia, 2011).

La metodología de relación de actividades es aplicable en problemas de distribución en instalaciones industriales, locales comerciales, hospitales, centros de distribución, entre otros. Entre los ejemplos se encuentran el caso del Centro de Distribución (CEDI) de Farmacéuticos "Aplicación de metodologías de distribución de plantas para la configuración de un centro de distribución" (Heidy Mejia, 2011). Así mismo, la empresa Natural S.A realizó un "Rediseño del sistema productivo utilizando técnicas de distribución de planta - Caso de estudio planta procesadora de alimentos" (Collazos Valencia, 2013). Con el fin de realizar una óptima distribución para el mejoramiento continuo de la empresa.

\footnotetext{
${ }^{6}$ Relación de Actividades o Relación de análisis de afinidades: Relación de cada departamento, oficina o área de servicios, con cualquier otro departamento y área.
} 


\section{MATERiales y MÉTOdoS}

En el caso de Alimentos Cárnicos S.A.S, el método relaciona los procedimientos encontrados en el área de proceso de separado ${ }^{2}$ y los califica según el nivel de importancia en la interacción de cada proceso para la elaboración del producto. En la Tabla 1 se presentan los valores de calificación de importancia y su significado para la relación de cada una de las actividades en dicha área.

TABla 1.

Calificación de importancia.

\begin{tabular}{|c|c|}
\hline Código & Definición \\
\hline A & $\begin{array}{c}\text { Absolutamente necesario } \\
\text { con 2 departamentos juntos }\end{array}$ \\
\hline E & Especialmente importante \\
\hline I & Importante \\
\hline O & Ordinariamente Importante \\
\hline U & Sin importancia \\
\hline $\mathrm{X}$ & No deseable \\
\hline
\end{tabular}

fuente: autores

Posteriormente, se realiza una aproximación de la distribución al identificar las actividades que se relacionan en el área de separado. La calificación de las actividades según el nivel de importancia y el orden adecuado del proceso se presentan en la Tabla 2.

TABLA 2.

Relación de Actividades

\begin{tabular}{|c|l|}
\hline ACTIVIDAD & \multicolumn{2}{|c|}{ RELACIONES ENTRE AREAS } \\
\hline 1 & HORNOS \\
\hline 2 & CARRITOS CON PRODUCTO \\
\hline 3 & CARRITOS SIN PRODUCTO \\
\hline 4 & MESA \\
\hline 5 & SEPARADORA \\
\hline 6 & ALAMACENAMIENTO PRODUCTO TERMINADO \\
\hline 7 & BANDA TRANSPORTADORA \\
\hline
\end{tabular}

Fuente: autores
Una vez se establecen las actividades y se califica su nivel de interacción, se procede a determinar el código de relación o de afinidad donde se establecen el grado de cercanía para los centros de actividades. El mencionado proceso se efectúa con el fin de realizar el diagrama adimensional de bloques que permite generar diferentes opciones de diseńo y se otienta a escoger el más óptimo.

Para garantizar que la relación del grado de cercanía sea relevante, coherente y se mantenga, se realiza un análisis de flujo en el diagrama adimensional de bloques. En la Figura 3 se presenta el diagrama adimensional que arrojó mejores resultados en cuanto a la interacción de las activades permitiendo un flujo constante y el espacio actual de la instalacion del area.

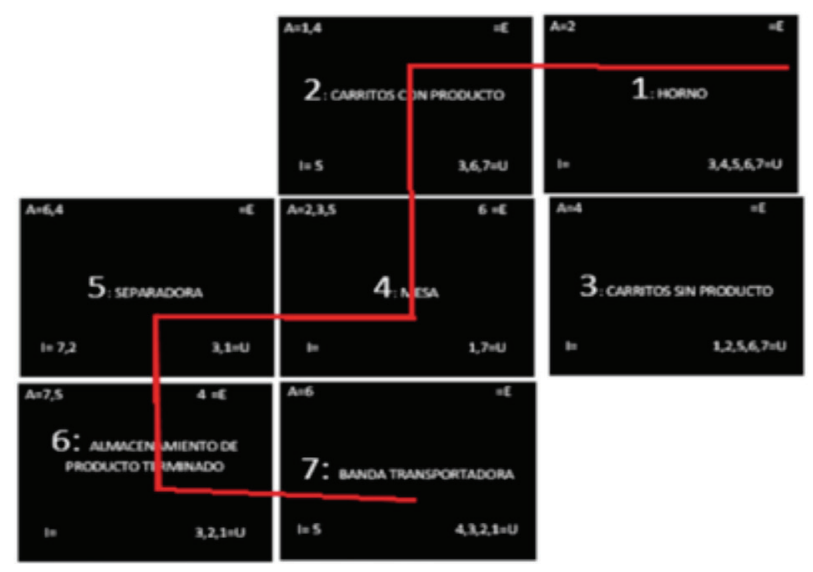

Fig. 3. Diagrama adimensional - Área de separado.

Al determinar el diagrama adimensional, se procede a diseñar el área de separado con la ayuda del software de diseño asistido por computador AutoCAD ${ }^{\circ}$ con el fin de realizar un estudio de las dimensiones de los equipos y de la instalación (Moreno, 2013).

En la Figura 4 se indica la propuesta de diseño en el área de separado. Se presenta el nuevo orden de los equipos, maquinas separadoras, mesas, canastillas y carritos y el recorrido de los operarios. 


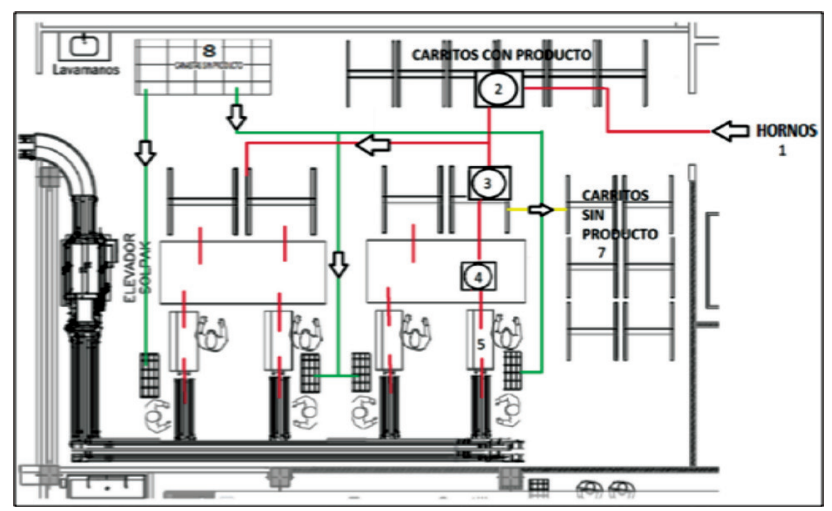

Fig. 4 Propuesta de distribución de planta en el área de separado diseñado en AutoCAD

\subsection{Simulación.}

Para realizar la simulación del proceso en el área de separado, se utilizó el Software Flexsim; que permite simular los procesos productivos industriales. Se consideró la información recolectada en las diferentes etapas del proceso, determinando los tiempos de cada máquina o equipos que interactúan con los operarios. Igualmente, se determinan las distancias que recorren los operarios en cada actividad, tanto su porcentaje de ocupación como el porcentaje de inactividad.

En el proceso del área de separado interactúan ocho operarios y cuatro máquinas en actividades distintas, como son Inotec 11, Towsend 10, Towsend 200, Towsend 13 (Alimentos Carnicos S.A.S, 2016) .

En la Tabla 3 se muestra las capacidades aproximadas de unidades por hora de cada una de las maquinas separadoras.

TABLA 3.

Capacidad máquinas separadoras

\begin{tabular}{|c|c|}
\hline SEPARADORA & CAPACIDAD $(\mathbf{U d s} / \mathbf{h})$ \\
\hline Inotec 11 & 22.799 \\
\hline Towsend 10 & 21.932 \\
\hline Towsend 200 & 22.978 \\
\hline Towsend 13 & 23.947 \\
\hline
\end{tabular}

fuente: autores

En la Figura 5 se ilustra el diseño actual del área de separado.

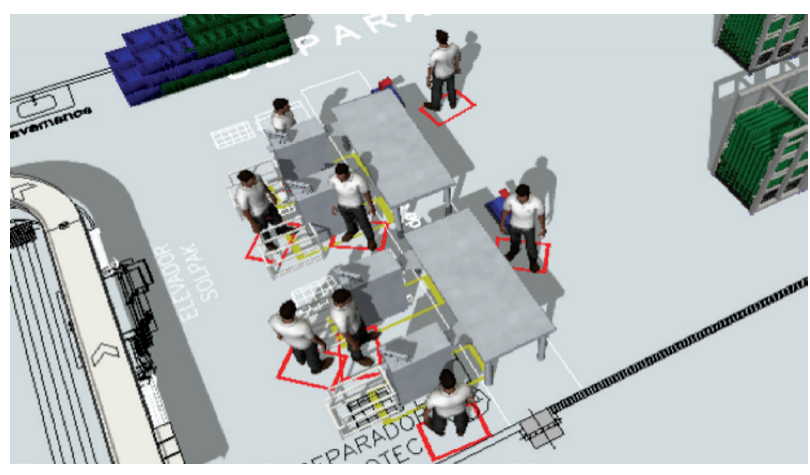

Fig. 5 Diseño actual del área de separado

\subsection{Descripción del modelo.}

Se procedió a validar el modelo en el software Flexsim. Para ello se utilizaron los siguientes objetos.

- Source. Son la fuente de entrada. En el modelo, los carros con los productos, las canastillas plásticas, los chorizos y salchichas entran por los source de acuerdo al programa de producción del día.

- Flowitems. Son los objetos que se mueven a través del modelo, para este caso los chorizos, las salchichas, las canastillas y los carros desplazables.

- Queu. Almacén o buffer de acumulación. Según el modelo, representan cada puesto donde los productos tienen que esperar para su siguiente proceso.

- Processor. Proceso o máquina. Representan cada una de las maquinas separadoras y su proceso. Se programan de acuerdo a la distribución de probabilidad bsada en el análisis de datos de la capacidad de producción de cada máquina. 
- Combiner. Se utiliza para agrupar dos o más Flowitems juntos mientras viajan a través de un modelo. Se utilizan para cargar los carros con los productos y empacar el producto terminado en las canastillas plásticas.

- Separator. Se utiliza para separar un Flowitem en múltiples partes. En este caso se utiliza para separar los productos de los carros.

- Conveyor. Banda o cinta transportadora, se utiliza para mover Flowitems por un camino determinado, en este caso se utilizó para simular la banda que lleva los productos desde el área de separado hasta el área de empaque.

\section{RESUlTADOS Y DisCUSION}

\subsection{Construcción del modelo de simulación.}

Se procede a remplazar gráficamente los objetos reales del área de separado, de tal manera que se programa el modelo de acuerdo al orden lógico del área de separado, como se observa en la Figura 6.

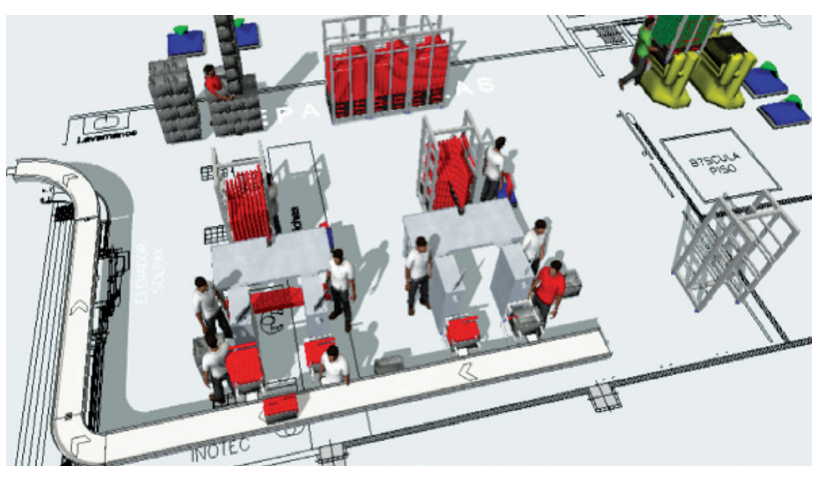

Fig. 6 Validación del modelo de simulación - software Flexsim.

\subsection{Variables a evaluar en el modelo.}

Una vez identificado el problema, se procede a evaluar las variables y ecuaciones presentadas en la Tabla 4 en el modelo propuesto.

TABla 4.

Fórmula matemática

\begin{tabular}{|c|c|c|c|}
\hline VARIABLES & UNIDAD & DESCRIPCIÓN & ECUACIÓN \\
\hline Tiempo de ocupación (To) & Horas & $\begin{array}{c}\text { Tiempo del operario } \\
\text { y la maquina operando. }\end{array}$ & To $=\sum T t-\sum T i$ (1) \\
\hline Tiempo inactivo (Ti) & Horas & $\begin{array}{c}\text { Tiempo del operario y la maquina } \\
\text { sin operatividad. }\end{array}$ & Do $=\sum R c+\sum R s \quad(3)$ \\
\hline Distancia recorrida (Do) & Horas & $\begin{array}{c}\text { Distancia de recorrido } \\
\text { por operario con carga }\end{array}$ & (2) \\
\hline Tiempo total (Tt) & Horas & $\begin{array}{c}\text { Tiempo total de operatividad del } \\
\text { sistema del proceso de producción. }\end{array}$ & $T t=\sum T o+\sum T i+\sum P p$ \\
\hline
\end{tabular}

Fuente: autores

\subsection{Escenarios de simulación.}

Para contrastar el diseño de planta actual con la propuesta planteada, se realizaron tres escenarios involucrando al personal del área de trabajo. En ellos se evaluó el porcentaje de ocupación, el porcentaje de inactividad y la distancia recorrida de cada operario en una jornada laboral.
TABLA 5.

Escenarios planteados

\begin{tabular}{|c|c|c|}
\hline Escenarios & Operarios & Separadoras \\
\hline 1 & 8 & 4 \\
\hline 2 & 10 & 4 \\
\hline 3 & 11 & 4 \\
\hline
\end{tabular}

Fuente: autores 


\subsection{Escenario actual.}

En el escenario 1 se simularon las condiciones actuales en el área de separado.
En la Tabla 6 se presenta el comportamiento de las variables respecto a la actividad que realizan los operarios.

TABLA 6.

Resultados Escenario1 (Condición actual) obtenidos por el simulador

\begin{tabular}{|c|c|c|c|c|}
\hline \multicolumn{5}{|c|}{ ACTIVIDAD DE OPERARIOS - DISTRIBUCIÓN ACTUAL } \\
\hline Uds. Procesadas & \multicolumn{3}{|c|}{$\mathbf{4 7 9 . 2 6 7}$} \\
\hline $\begin{array}{c}\text { Tiempo total } \\
\text { (horas) }\end{array}$ & $\mathbf{1 2 , 8 3}$ & \multicolumn{3}{|c|}{ 7:00 AM - 7:50 PM } \\
\hline Operario & Ocupación (\%) & Inactivo (\%) & Distancia (km) & Salida \\
\hline Oper 1 & 42,08 & 57,92 & 10,5 & 4619 \\
\hline Oper 2 & 38,66 & 61,34 & 7,8 & 4597 \\
\hline Oper 3 & 51,10 & 48,90 & 1,3 & $\ldots-$ \\
\hline Oper 4 & 53,00 & 47,00 & 1,1 & $\ldots$ \\
\hline Oper 5 & 48,00 & 52,00 & 1,1 & $\ldots$ \\
\hline Oper 6 & 47,00 & 53,00 & 1,2 & -- \\
\hline Oper 7 & 37,84 & 62,16 & 12,9 & 2102 \\
\hline Oper 8 & 35,01 & 64,99 & 8,6 & 2026 \\
\hline
\end{tabular}

fuente: autores

En la Tabla 6 se evidencia que para procesar 479.267 unidades de chorizo se requiere de 12.83 horas de trabajo, incluyendo en ese tiempo las paradas programadas. En el mencionado escenario, el operario 1, encargado de llevar las canastas con producto, hace un recorrido de 10.5 kilómetros aproximadamente, realizando la misma actividad 4.619 veces, con un porcentaje de ocupación del $42.08 \%$ y un porcentaje ocio del $57.92 \%$. Así mismo el operario 2, encargado de llevar las canastas con producto, hace un recorrido de 7.8 kilómetros aproximadamente, realizando la misma actividad 4.597 veces, con un porcentaje de ocupación del $38,66 \%$ y un porcentaje ocio del 61,34\%.
La distancia recorrida por los operarios 3, 4, 5 y 6 no es significativa, debido a que ellos están encargados de operar las separadoras y de inspeccionar el producto. Para ellos, el porcentaje de ocupación se encuentra entre $47 \%$ y $53 \%$.

Por otra parte, se identificó que el operario 7, encargado de llevar los carros con producto hacia la mesa, presenta la mayor distancia recorrida dentro del proceso de separado. Una distancia total recorrida de 12.9 kilómetros realizando la misma actividad 2.102 veces, con un porcentaje de ocupación del $37.84 \%$ y un porcentaje de tiempo ocio de $62.16 \%$. Los resultados se presentan gráficamente en la Figura 7. 


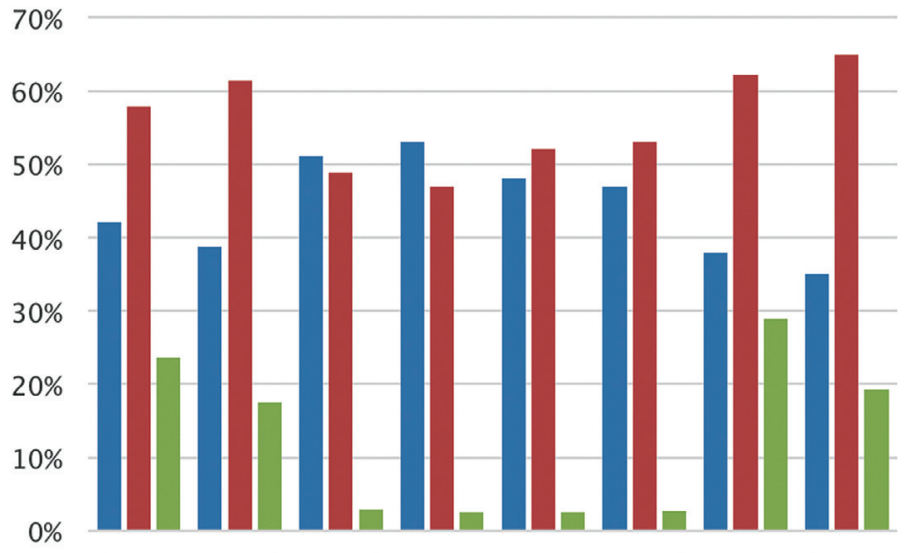

Oper 1 Oper 2 Oper 3 Oper 4 Oper 5 Oper 6 Oper 7 Oper 8

\subsection{Escenario 2: mejora 1.}

En el escenario 2 se realiza la simulación con el diseño de planta propuesto en el área de separado con 10 operarios para analizar el comportamiento del sistema bajo estas condiciones e identificar si disminuye la carga laboral de los operarios. En este entorno se mantuvo constante el tiempo total y la cantidad de unidades procesadas. En la Tabla 7 se presentan los resultados de la simulación.

\section{\% OCUPACION \% INACTIVO DISTANCIA}

Fig. 7 Resultados Escenario 1.

TABLA 7.

Resultados Escenario 2 (10 operarios)

\begin{tabular}{|c|c|c|c|c|c|}
\hline \multicolumn{6}{|c|}{ ACTIVIDAD DE OPERARIOS - ESCENARIO 2} \\
\hline \multicolumn{2}{|c|}{ Uds. Procesadas } & \multirow{2}{*}{\multicolumn{3}{|c|}{$\begin{array}{c}479.267 \\
7: 00 \mathrm{AM}-7: 50 \mathrm{PM}\end{array}$}} & \multirow{3}{*}{$\begin{array}{l}\text { Diferencia } \\
\text { Distancia }\end{array}$} \\
\hline Tiempo total (horas) & 12,83 & & & & \\
\hline Opera & $\%$ Ocu & $\%$ Inac & Dist $(\mathbf{k m})$ & Salida & \\
\hline Oper 1 & 42,08 & 57,92 & 9,4 & 4619 & 1,1 \\
\hline Oper 2 & 38,66 & 61,34 & 6,6 & 4597 & 1,2 \\
\hline Oper 3 & 57,30 & 42,70 & 1,3 & --- & 0 \\
\hline Oper 4 & 55,30 & 44,70 & 1,1 & --- & 0 \\
\hline Oper 5 & 47,30 & 52,70 & 1,1 & --- & 0 \\
\hline Oper 6 & 48,10 & 51,90 & 1,2 & -- & 0 \\
\hline Oper 7 & 37,84 & 62,16 & 1,3 & 867 & 11,6 \\
\hline Oper 8 & 42,63 & 57,37 & 18,8 & 1992 & $-10,2$ \\
\hline E 1 & 40,17 & 59,83 & 20,4 & 3455 & 20,4 \\
\hline E 3 & 31,61 & 68,39 & 3,9 & 868 & 3,9 \\
\hline
\end{tabular}

*E1: Operario Extra 1

*E3: Operario Extra 3

En el escenario 2, el operario E1 está encargado de trasladar las canastillas a las separadoras, realizando un recorrido total de 20.4 kilómetros equivalentes a porcentaje de ocupación del $40.17 \%$ y $59.83 \%$ de tiempo ocio.
El operario E3 está encargado de trasladar las canastillas con los chorizos hacia la banda transportadora. Él registra un porcentaje de inactividad del $68.39 \%$, que es uno de porcentajes de ocio más altos identificados en la simulación. Esto se debe 
a la falta de constancia en su proceso. El operario E3 recorre 3.9 kilómetros en total y cuenta con un $31.61 \%$ de ocupación.

El operario 1 reduce su recorrido de 10,5 kilómetros en la distribución actual, a 9,4 kilómetros en el escenario 2, disminuyendo 1,1 kilómetros. Para el operario 2, quien realiza la misma acción, la simulación registra una reducción en la distancia de recorrido, de 7,8 kilómetros en la condición actual a 6,6 kilómetros en el escenario 2, con una diferencia de 1,2 kilómetros.

El operario 7, quien cumple con transportar los carros hacia la mesa que salen del área de enfriamiento, tuvo una reducción de recorrido de 11,6 kilómetros. El operario 8, quien está en dos separadoras, resulta con un porcentaje de ocupación del 42,63\%, $57.37 \%$ de ocio, y realiza un recorrido de 18,8 kilómetros, aumentando en 10,2 kilómetros la distancia recorrida. Los resultados se presentan gráficamente en la Figura 8.

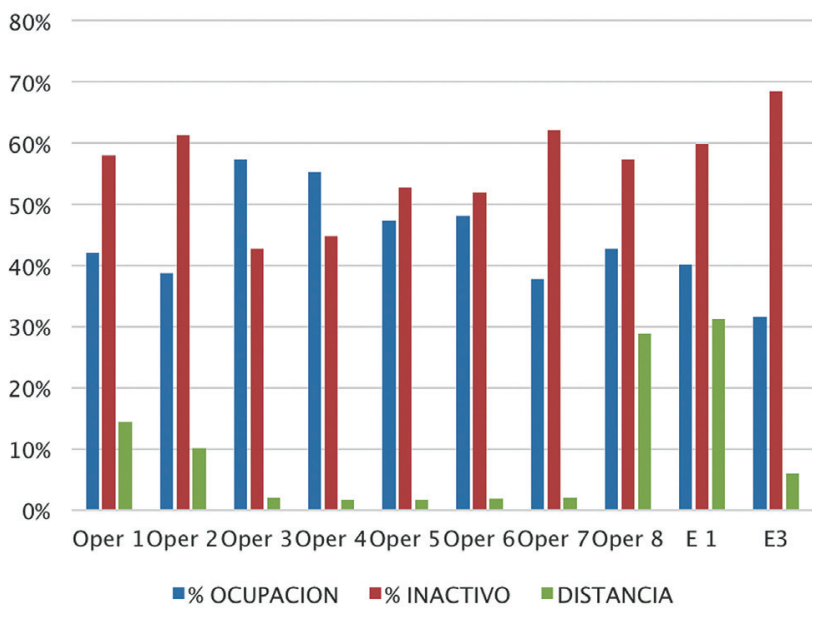

Fig. 8 Resultados Escenario 2

\subsection{Escenario 3: mejora 2.}

En el escenario 3, se simula el modelo con un operario adicional, con el fin de identificar el comportamiento y determinar los efectos en la reducción de la carga laboral de los operarios. En este entorno se mantuvo constante el tiempo total y la cantidad de unidades procesadas. En la Tabla 8 presentan los resultados de la simulación del escenario 3 .

TABla 8.

Resultados escenario 3 (11 operarios)

\begin{tabular}{|c|c|c|c|c|c|}
\hline \multicolumn{6}{|c|}{ ACTIVIDAD DE OPERARIOS - ESCENARIO 3} \\
\hline \multicolumn{2}{|c|}{ Uds. Procesadas } & \multirow{2}{*}{\multicolumn{3}{|c|}{$\begin{array}{c}479.267 \\
7: 00 \mathrm{AM}-7: 50 \mathrm{PM}\end{array}$}} & \multirow{3}{*}{$\begin{array}{l}\text { Diferencia } \\
\text { Distancia }\end{array}$} \\
\hline \multirow{2}{*}{$\begin{array}{c}\text { Tiempo total (horas) } \\
\text { Opera } \\
\end{array}$} & \multirow{2}{*}{$\begin{array}{c}12,83 \\
\% \text { Ocupa }\end{array}$} & & & & \\
\hline & & $\%$ Inac & Dist (km) & Salida & \\
\hline Oper 1 & 42,08 & 57,92 & 6,6 & 867 & 3,9 \\
\hline Oper 2 & 38,66 & 61,34 & 9,4 & 1992 & $-1,6$ \\
\hline Oper 3 & 35,30 & 64,70 & 1,3 & -.- & 0 \\
\hline Oper 4 & 36,80 & 63,20 & 1,1 & -- & 0 \\
\hline Oper 5 & 49,60 & 50,40 & 1,1 & -- & 0 \\
\hline Oper 6 & 47,30 & 52,70 & 1,2 & --- & 0 \\
\hline Oper 7 & 37,84 & 62,16 & 1,3 & 867 & 11,6 \\
\hline Oper 8 & 35,01 & 64,99 & 2,3 & 870 & 6,3 \\
\hline E 1 & 40,17 & 59,83 & 20,4 & 3455 & 20,4 \\
\hline E 3 & 24,17 & 75,83 & 2,3 & 868 & 2,3 \\
\hline E4 & 31,61 & 68,39 & 3,9 & 871 & 3,9 \\
\hline
\end{tabular}


La distancia recorrida por el operario E1 y el porcentaje de ocupación se mantuvieron constantes entre el escenario 2 y 3 . Para el operario E3, que recibe el producto en las canastillas y las ubica en la banda transportadora, se reduce la distancia recorrida en 1.6 kilómetros. Para el operario 8 se redujo en 6,3 kilómetros la distancia recorrida.

Los operarios 3, 4, 5 y 6 , al igual que en los anteriores escenarios, no presentan diferencias en cuanto a las distancias recorridas entregadas por el simulador, debido que ellos son los operarios encargados de las maquinas separadoras y no requieren de hacer mayores desplazamientos, sin embrago, si presentan variaciones en los tiempos de ocupación e inactividad como se presenta en la Figura 9.

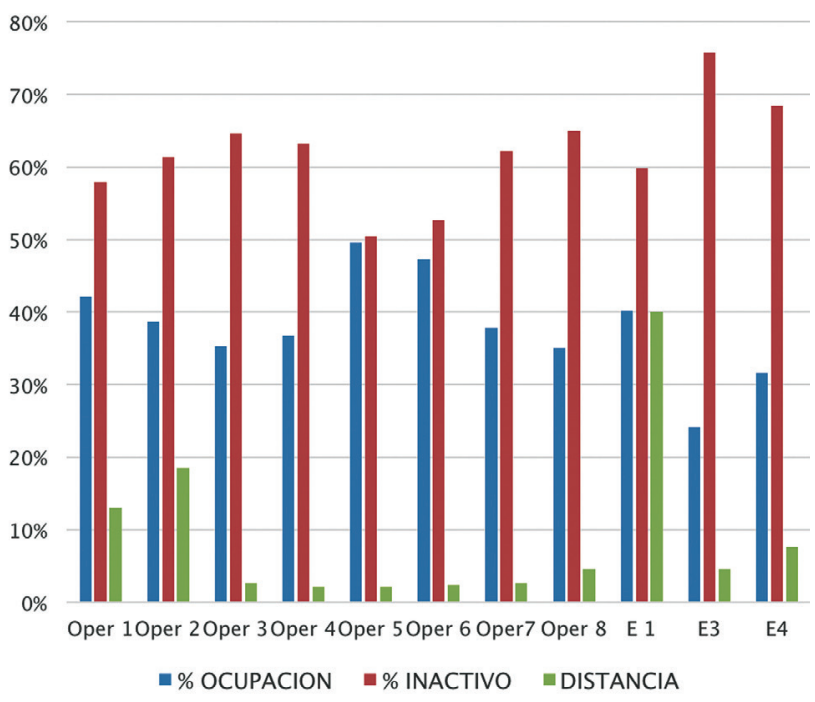

Fig. 9 Resultados Escenario 3.

De acuerdo al estudio realizado y la comparación de las Figuras 7, 8 y 9, se determinó que escenario que arroja mejores resultados en cuanto a seguridad y salud en el trabajo y gestión de recursos humanos es el escenario 2. Esta selección se basó en la norma técnica colombiana NTC 5693-1 donde se especifican los límites recomendados para el levantamiento y transporte manual considerando la intensidad, frecuencia y duración de la actividad.

En el escenario 2, los operarios que presentan mayor frecuencia de recorrido y mayor distancia, mantienen un umbral menor al presentado en los otros escenarios. Lo que reduce considerablemente el riesgo para la salud y aumenta la seguridad del recurso humano como se presenta en el siguiente análisis de los operarios de mayor impacto.

En el caso del operario 1 y operario 2 , se presentó una disminución de 1.1 kilómetros de distancia aproximadamente en cada uno. La operación realizada tiene una frecuencia de repeticiones de 4.619 veces equivalente a 43.87 toneladas de carga total para los operarios 1 y 2. Esto se ve reflejado, a su vez, en el porcentaje de ocupación y el de inactividad (ver Tabla 7).

En el caso del operario E1, encargado de llevar las canastillas vacías hacia las separadoras y quien recorre la mayor distancia en la simulación, presenta un recorrido total de 20,4 kilómetros, con una actividad repetitiva de 3.455 veces, que equivale a $\mathbf{1 . 1}$ tonelada de carga total. No obstante, el porcentaje de inactividad es uno de los más altos de la simulación, con 59.83\%. Lo anterior se debe principalmente a que su actividad no tiene una constancia determinada.

Adicionalmente, el operario 8, quien debe estar en dos separadoras, realiza un recorrido de 18,8 kilómetros. Recorrido que aumentó en 10,2 kilómetros en relación con el primer escenario. El operario 8 realiza una actividad repetitiva de 1.992 veces, trasladando una carga total de $\mathbf{3 8}$ toneladas, que equivale al $22,88 \%$. La reducción de distancia recorrida, observada al simular el tercer escenario, se debe a la restricción de funcionalidades del operario 8 en relación con la operación de las dos separadoras.

\section{Conclusiones y trabajos futuros}

La optimización del proceso se centra en la seguridad y salud en el trabajo. Específicamente en el incremento de la seguridad de los operarios y en la reducción del riesgo para la salud. La distribución óptima de los equipos y áreas presentada en este trabajo garantiza un incremento en la seguridad en el proceso de separado 
en la empresa de Alimentos Cárnicos al reducir considerablemente las distancias recorridas por los operarios manteniendo un buen porcentaje de ocupación en los mismos. De igual forma, presenta reducciones significativas en los cuellos de botella.

La simulación de los escenarios propuestos permite representar un sistema real, evaluando variables que permiten cumplir con el desempeño del objetivo de la empresa. La simulación aportó a esta investigación una reducción considerable de tiempo de análisis y corroboró el escenario 2 como el más eficiente donde se redujo el tonelaje y los kilómetros recorridos en aproximadamente un 10\%. Así mismo el porcentaje de actividad se incrementó en $0,12 \%$ en promedio para los operarios en el escenario 2 .

Este proceso de investigación evidencia la importancia de la simulación para la toma de decisiones en distribución en planta debido a que permite evaluar variables como: carga laboral, frecuencia de realización de actividades por tarea y distancias. La herramienta refleja el comportamiento del modelo en tiempo real para una mejora continua de los procesos industriales. Igualmente, la simulación facilitó la presentación de los resultados a los jefes de planta, incorporando animaciones en 3D que permiten evaluar y presentar los procesos.

Se propone, como trabajos futuros, el análisis de otros procesos complementarios en la planta de producción de Alimentos Cárnicos S.A.S con el fin de evaluar las variaciones de tiempos y distancias asociadas a la integración entre los mismos, aumentando el grado de complejidad y brindando análisis más completos y cercanos a la realidad.

\section{REFERENCIAS}

Alimentos Carnicos S.A.S. (2016). Capacidad de produccion de maquinas separadoras. Guachene - Cauca.

ARL SURA. (17 de Julio de 2008). Resolución 002646 de julio de 2008. Recuperado el 30 de Marzo de 2018, de https://www. arlsura.com/index.php/decretos-leyes-resoluciones-circularesy-jurisprudencia/206-resoluciones/1177-resolucion-numero2646-de-2008
Bryan, S. L. (31 de Mayo de 2016). Ingenieria industrial online. com. (DISEÑO Y DISTRIBUCIÓN DE PLANTA) Recuperado el 24 de Marzo de 2018, de https://www.ingenieriaindustrialonline.com/herramientas-para-el-ingeniero-industrial/ dise $\%$ C3\%B1o-y-distribuci\%C3\%B3n-en-planta/

Caputo, A. C., Pelagagge, P. M., Palumbo, M., \& Salini, P. (2015). Safety-based process plant layout using genetic algorithm. Journal of Loss Prevention in the Process Industries, 139-150.

Collazos Valencia, C. J. (2013). Rediseño del sistema productivo utilizando técnicas de distribución de planta: Caso de estudio planta procesadora de alimentos. Doctoral dissertation, Universidad Nacional de Colombia-Sede Manizales, 9-84.

Diego-Más, J. A. (2006). Optimización de la distribución en planta de instalaciones industriales mediante algoritmos genéticos. Aportación al control de la geometría de las actividades. Memoria de la Tesis Doctoral. UPV. Valencia, España., 6-445.

Grupo Empresarial Nutresa S.A. (2010). Alimentos Carnicos S.A.S. (Historia) Recuperado el 24 de Abril de 2018, de http://www. alimentoscarnicos.com.co/index.php/informacion-institucional/nuestra-historia

Grupo Empresarial Nutresa S.A. (2010). Alimentos Carnicos S.A.S. Recuperado el 24 de Marzo de 2018, de http://www.alimentoscarnicos.com.co/index.php/informacion-institucional/nuestra-historia.

Heidy Mejia, M. J. (2011). Aplicación de metodologías de distribución de plantas para la configuración de un centro de distribución. Barranquilla Colombia.

ICONTEC Internacional. (2009). Ergonomia, manipulación manual. Parte 1: Levantamiento y transporte. Bogota.

Khiewwijit, R., Temmink, H., Rijnaarts, H., \& Keesman, K. J. (2015). Energy and nutrient recovery for municipal wastewater treatment: how to design a feasible plant layout? Environmental Modelling \& Software, 156-165.

Khuriyati, N., Sukartiko, A. C., \& Kartikasari, A. (2016). Plant Layout for Small Scale Food Industry. KnE Life Sciences, 98-103.

Moreno, L. (22 de Abril de 2013). Blog de Informatica. Recuperado el 24 de Marzo de 2018, de http://leonardoqta03.blogspot. com.co/2013/04/que-es-autocad-y-para-que-nos-sirve.html

Muther, R. (1981). Distribución en planta. McGraw Hill.

Practicas de sistemas de fabricación. (6 de Febrero de 2012). Automatica y Robotica. Recuperado el 24 de Marzo de 2018, de https://rua.ua.es/dspace/bitstream/10045/20587/1/Simulacion_de_un_proceso_industrial_mediante_FlexSim.pdf

Stephens, F. E.-M. (2006). Analisis de la relación de Actividades. En Diseño de instalaciones de manufactura y manejo de materiales. (págs. 180-181). Bogota: PEARSON.

Vargas, A. Z., Vargas, L. S., \& Franco, J. A. (2018). Diseño de planta piloto para la obtención de plata granalla utilizando un sistema de electrodeposición cilíndrico cerrado. Nexo Revista Cientifica, 111-122. 
